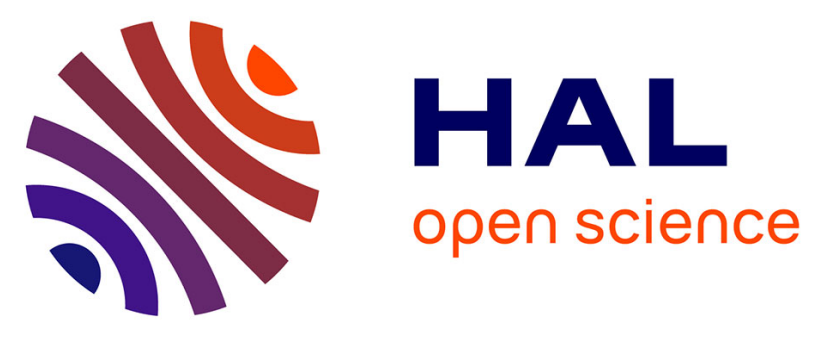

\title{
A methodology to benchmark flexible payload architectures in a megaconstellation use case
}

Florian Vidal, Hervé Legay, George Goussetis, Maria Garcia Vigueras, Segolene Tubau, Jean-Didier Gayrard

\section{- To cite this version:}

Florian Vidal, Hervé Legay, George Goussetis, Maria Garcia Vigueras, Segolene Tubau, et al.. A methodology to benchmark flexible payload architectures in a megaconstellation use case. International Journal of Satellite Communications and Networking, 2021, 39 (1), pp.29-46. 10.1002/sat.1344 . hal-02530973

HAL Id: hal-02530973

https://hal-univ-rennes1.archives-ouvertes.fr/hal-02530973

Submitted on 7 Apr 2020

HAL is a multi-disciplinary open access archive for the deposit and dissemination of scientific research documents, whether they are published or not. The documents may come from teaching and research institutions in France or abroad, or from public or private research centers.
L'archive ouverte pluridisciplinaire HAL, est destinée au dépôt et à la diffusion de documents scientifiques de niveau recherche, publiés ou non, émanant des établissements d'enseignement et de recherche français ou étrangers, des laboratoires publics ou privés.

\section{(c)(1)}

Distributed under a Creative Commons Attribution| 4.0 International License 


\title{
A methodology to benchmark flexible payload architectures in a megaconstellation use case
}

\author{
Florian Vidal ${ }^{1}$ | Hervé Legay ${ }^{1}$ | George Goussetis ${ }^{2}$ | Maria Garcia Vigueras ${ }^{3}$ | \\ Ségolène Tubau $^{1} \mid$ Jean-Didier Gayrard ${ }^{1}$
}

${ }^{1}$ Thales Alenia Space, BP 1187, 31037 , Toulouse Cedex 1, France

${ }^{2}$ Institute of Sensors, Signals \& Systems, Heriot-Watt University, Edinburgh, United Kingdom

${ }^{3}$ IETR Laboratory, INSA Rennes, 20 Avenue des Buttes de Coesmes, 35700 Rennes, France

\section{Correspondence}

Florian Vidal, Thales Alenia Space, BP 1187, 31037 Toulouse Cedex 1, France.

Email: florian.vidal@thalesaleniaspace.com

Funding information H2020 Marie Skłodowska-Curie Actions, Grant/Award Number:

MSCA-ITN-2016-722840

\begin{abstract}
Summary
This paper proposes a methodology to benchmark satellite payload architectures and find the optimal trade-offs between high flexibility and low complexity. High flexibility would enable the satellite to adapt to various distributions of user terminals on the ground and fulfill the data rate demand of these users. Besides, low complexity is required to keep satellite networks competitive in the context of emerging $5 \mathrm{G}$ networks. To estimate the flexibility of a payload, an indicator to characterize the non-uniformity of user distributions is proposed. Each benchmarked payload may be characterized by a graph relating the throughput to this parameter further denoted $\mu$. The payload provides the same throughput trends for different scenarios of user distributions with the same $\mu$ parameter. As a consequence, the average capacity of the system may be estimated by (a) calculating the probability distribution of $\mu$ over the orbit and (b) integrating the throughput based on this payload response. It thus results in a straightforward way for benchmarking payloads directly on an estimation of the averaged capacity, accounting for the user distribution over the earth. A simulation platform has been developed to characterize the payload throughput including the implementation of a resource allocation algorithm that accounts for constraints of various payloads. Using this definition and the developed tool, we benchmark a bent-pipe architecture, a beam hopping architecture and a hybrid beam-steering architecture for a LEO megaconstellation use case. The methodology showcases the interest for investigating different payload architectures depending on realistic traffic scenario analysis.
\end{abstract}

\section{KEYWORDS}

hybrid beamforming, LEO, megaconstellation, multibeam, payload, resource allocation

\section{1 | INTRODUCTION}

Traditional satellite telecommunication networks are based on GEO satellites and offer either mobile or fixed services that exploit the wide coverage offered by satellite platforms. ${ }^{1}$ These services are based on broadcast, multicast, or unicast. Broadcast is used for radio or TV services, whereas multicast and unicast are more utilized for data services, mobile communications, and broadband internet and multimedia services. ${ }^{2}$ Broadband services require high data rates per user terminal and consequently wide frequency bands and high Equivalent Isotropic Radiated Power (EIRP) to close the link budget. To meet this challenge, satellites implement antenna architectures based on multibeam coverages with high antenna directivities to ensure the EIRP requirements and frequency reuse to increase the aggregated frequency bandwidth available. ${ }^{3}$ Considering satellites in a $5 G$ environment, other constraints such as latency, wide coverage and reliability. In this context, LEO satellite megaconstellations bring promising advantages compared with GEO satellites:

- The round-trip time latency for a GEO satellite is 500 milliseconds because of the $36000 \mathrm{~km}$ path between the satellite and Earth, which is far from the 1-millisecond objective of 5G. ${ }^{4}$ This requirement is crucial for real time health monitoring or finance transactions for example. ${ }^{4}$ For a satellite at $1200-\mathrm{km}$ altitude, this round-trip latency is reduced to 16 milliseconds, which makes them a more viable solution than GEO satellite for applications requiring low latency.

This is an open access article under the terms of the Creative Commons Attribution License, which permits use, distribution and reproduction in any medium, provided 
- A constellation of non-GEO satellites may, in the case of a polar orbit for example, offer a seamless quality of service even at the Earth poles, which is more challenging for GEO satellites because of low user elevations at these latitudes.

- The high number of satellites in megaconstellations makes the loss of a satellite not critical for the complete system (graceful degradation of the service). Moreover, spare satellites may be placed in orbit in case of failure at low cost compared with the overall system CAPEX.

The previous points emphasize that LEO megaconstellations seem to be a competitive solution to support $5 \mathrm{G}$ networks. LEO satellites experience very different traffic distributions in their antenna field of views (FOVs) as they orbit around the Earth. At payload level, introducing flexibility is of interest to address user distributions that may range from uniform over all the FOV (Figure $1 \mathrm{~A}$ ) to very dense concentrations, corresponding for example to scenarios with hubs like cities (Figure 1B). In a uniform scenario, a uniform repartition of resources among beams is optimal to meet the user demands. This solution can be provided by bent-pipe payloads associated with non-steerable multiple beam antennas. This approach was selected by OneWeb with its bent-pipe satellites with 16 non-steerable, highly elliptical beams. ${ }^{5}$ However, in an uneven scenario, only a limited number of the beams are potentially active, so flexibility in resource allocation is essential to meet all the demand. In that case, no flexibility would both waste resources over non-populated areas and be insufficient to match the throughputs required over densely populated areas. Satellite operators would then reduce the data rates proposed to their customers and lose potential earnings. Flexibility necessitates more complex payloads including for example beam hopping $(\mathrm{BH})$, inter-satellite links, or reconfigurable antennas. This technological choice was followed by Telesat and SpaceX Starlink megaconstellations. ${ }^{5}$

Flexibility at payload level enables the satellites to reallocate their resources in power, frequency, time, or coverage to adapt to these various scenarios. Beam hopping, whereby satellite resources are shared in the time domain, is increasingly being considered as an attractive solution. ${ }^{6}$ It consists in splitting time in slots and illuminating a set of beams at each time slot. Ferrite switches is a mature technology for the implementation of $\mathrm{BH}^{7}$ In the spatial domain, flexibility by virtue of beam steering may be achieved with mechanically steerable antennas or phased arrays. Phased array solutions require a Beam-forming network (BFN) that can be done either digitally in the Digital Transparent Processor (DTP) in the digital core, ${ }^{8}$ or in an analog way, for example, with phase shifters, Butler matrices, or quasi-optical beam formers (QOBFs). ${ }^{9}$ Hybrid beam forming strategies combining analog and digital techniques can limit the complexity of the BFN. ${ }^{10}$ There is thus a wide range of solutions to implement flexibility in satellite payloads, and there is consequently a need for benchmarking these solutions in performances and in complexity.

In the following, a methodology to compare payload architectures taking into account the different domains of flexibility is presented. This methodology has at its core a modular tool that implements a resource allocation algorithm. A parameter to characterize the non-uniformity of user distributions is introduced. Thanks to this parameter, the ability of various LEO payload architectures to support different scenarios of user distributions that may be encountered along their orbits is assessed. To illustrate the benefits of this methodology, different LEO satellite payload architectures with different levels of flexibility and complexity are benchmarked with a focus on the forward downlink throughput between the satellite and the user terminals. These architectures are first described in Section 3. An approach to parameterize payload designs is presented in Section 4. The non-uniformity parameter and the resource allocation algorithm used to compute throughputs are described Section 5; finally, results are presented in Section 6.

\section{2 | ASSESSED PAYLOADS}

A megaconstellation scenario with polar orbiting satellites at $1200-\mathrm{km}$ altitude is assumed for hosting all the considered payloads. The satellites operate in the Ka band to access more frequency bandwidth and provide broadband services. One particular issue with LEO satellites is the difference in attenuation experienced between the nadir and the edge of coverage due to increasing path losses. In this context, path losses reflect the RF signal attenuation as it propagates in the atmosphere. They may be caused by the expansion of the wave front in free space (free space loss, defined in Equation (1) with $d$, the distance between the satellite and the user terminal and $\lambda$ the wavelength) or by absorption (rain, clouds, gazes). Here, a clear sky scenario is considered so just the free space loss as defined in (1) is taken into account. In this context, FOV is

FIGURE 1 Antenna field of view, the beams used are filled, users are represented by dots. A, Uniform user distribution; B, non-uniform user distribution [Colour figure can be viewed at wileyonlinelibrary.com]
(A)

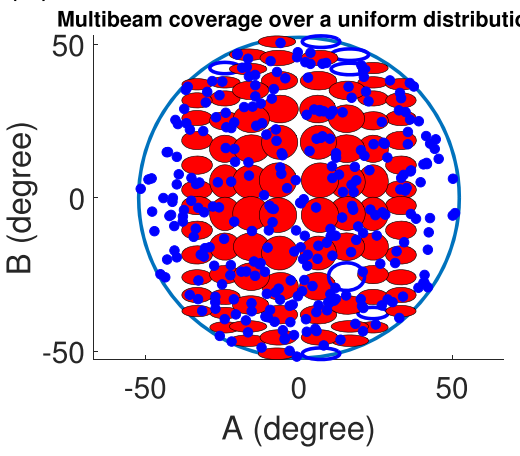

(B)

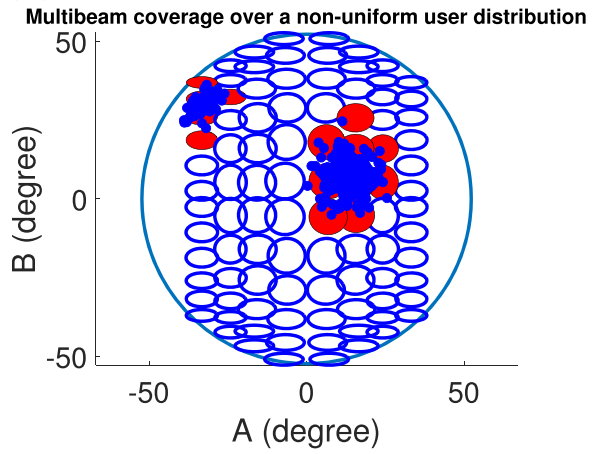


defined as the region where users have the satellite in line-of-sight with over $20^{\circ}$ of elevation angle. Under this value, satellites may be masked by building or mountains. Furthermore, the link budgets are poorer due to higher distances between the satellite and the user terminals.

$$
\text { FreeSpaceLoss }=\left(\frac{4 \pi d}{\lambda}\right)^{2}
$$

The variation of free space loss as a function of the elevation angle is presented in Figure $2 \mathrm{~A}$. In the case of $20^{\circ}$ of elevation for a user on the ground, the user is at a scan angle of $52^{\circ}$ that corresponds to an extra path loss of $6.2 \mathrm{~dB}$.

To provide a uniform EIRP over the coverage, more directive beams are needed to compensate for increased path losses at high elevations. This type of coverage, an example of which is schematically depicted in Figure 2B, is referred to as isoflux because it ensures an almost constant power flux on the ground. It is noted that the projection of the beams of an isoflux coverage on the surface of the Earth results in comparable footprints. With reference to Figure 2B, this suggests that due to the Earth's curvature and the pointing angle, beams at the edge of the coverage would have larger footprints than if they were pointed at nadir.

Given these comparable footprints, over multiple orbits, all beams would experience similar maximum traffic per beam. Moreover, coordination constraints with users of GEO satellites systems suggest steep beam slopes along the north-south axis for reducing interferences; consequently, beams with elliptical shapes were specified. With such a coverage based on elliptical beams, the operator of satellites in polar orbit can switch off some beams (to avoid interference from the ground to the GEO satellites) with less impact on the users in the north-south direction.

In the remaining sections, coverages are represented in AB coordinates, which satisfy

$$
\begin{aligned}
\theta & =\sqrt{A^{2}+B^{2}} \\
\phi & =\arctan (A / B) .
\end{aligned}
$$

In Equations (2), $\theta$ and $\phi$ represent the spherical coordinates in the satellite coordinate system; the $Z$ axis as represented in Figure 3 is pointing toward Earth, and the $Y$ axis is in the direction of the satellite's velocity.

\section{1 | Multiple fixed beam coverage with bent-pipe transponders}

An antenna solution to meet the isoflux requirement is the antenna concept shown in Figure $4 \mathrm{~A}$ and described in Tubau et al ${ }^{11}$ based on $\mathrm{QOBF}$ and cylindrical reflectors. This antenna architecture produces several beams from the same radiating aperture and is therefore a compact solution

(A)
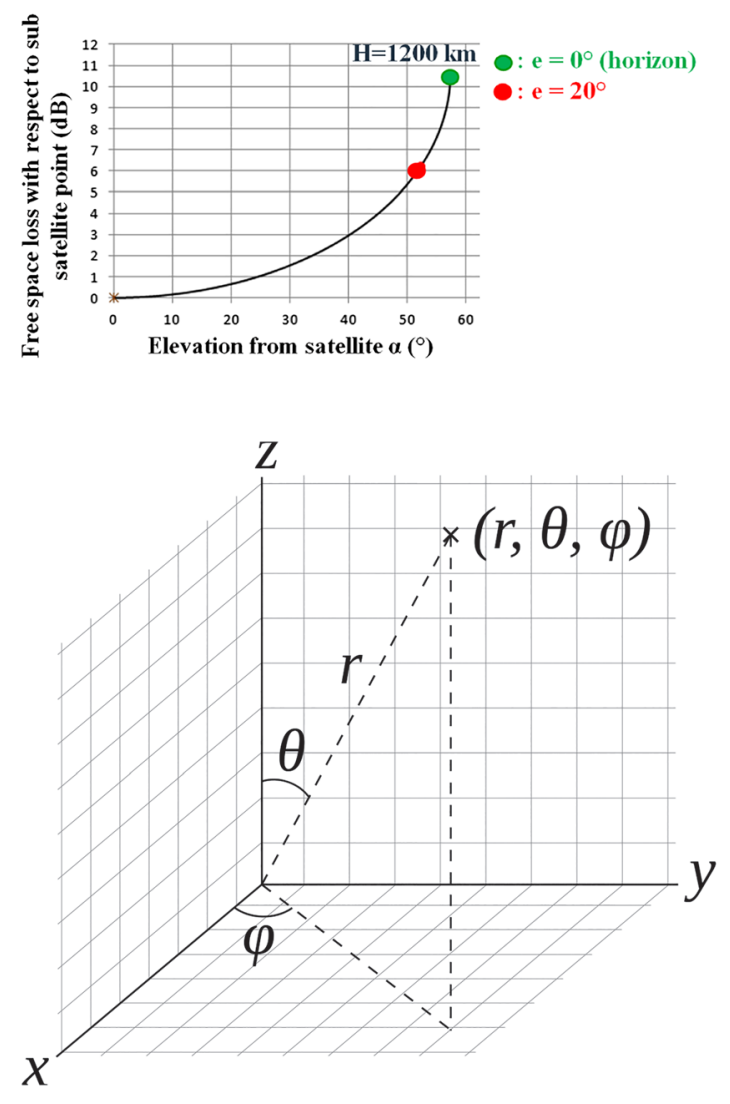

(B)

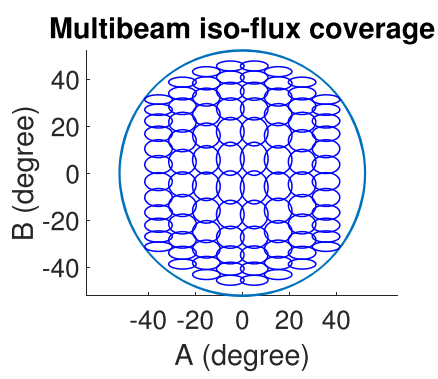

FIGURE 2 A, Free space loss for users at high elevations at $1200-\mathrm{km}$ altitude; B, Multiple beam coverage with the isoflux characteristic formed by 96 beams along eight columns. The ellipses represents the 3- $\mathrm{dB}$ contour of the beams [Colour figure can be viewed at wileyonlinelibrary.com] 
(A)

FIGURE 4 A, Quasi-optical beam formers with cylindrical reflector; $B$, accommodation of the whole antenna set up on a small satellite platform [Colour figure can be viewed at wileyonlinelibrary.com]

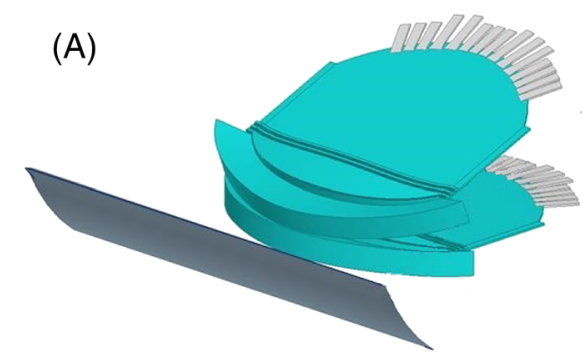

(A)

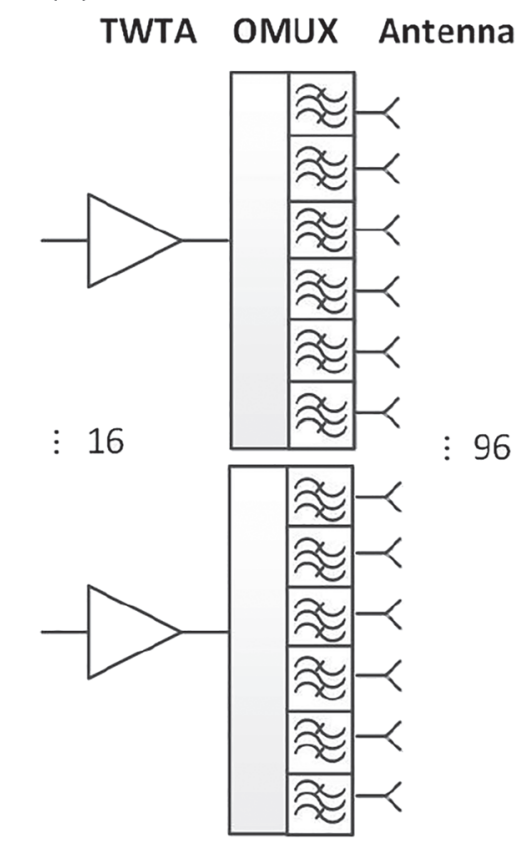

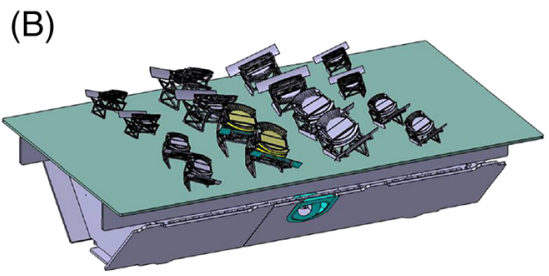

(B)

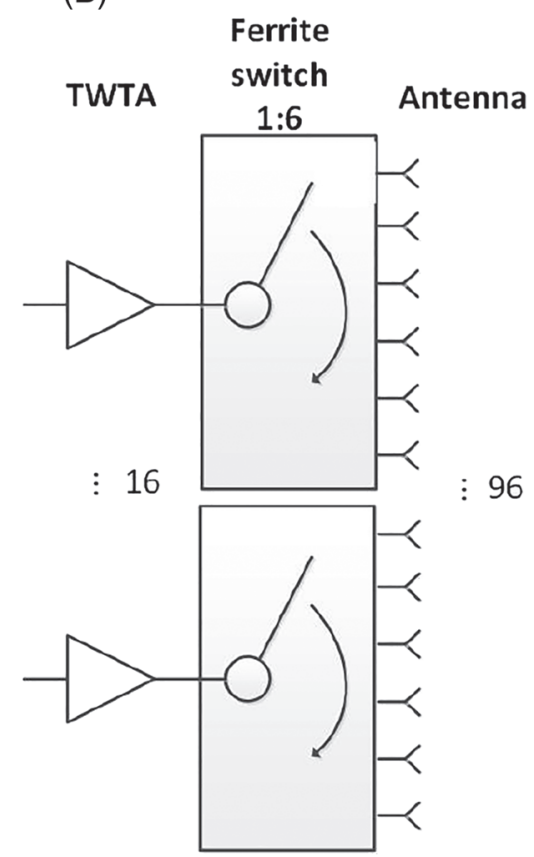

architecture; B, beam hopping architecture

for current LEO satellite platforms. The accomodation constraint took into account 17 satellite platforms fit in a 5-m diameter launcher fairing, which imposes a maximum volume of $1.5 \times 5 \times 0.5 \mathrm{~m}$ for the antenna farm. ${ }^{11}$ Figure 4B shows the accommodation of the Tx and Rx antennas on a small platform suitable for allowing multiple satellites per launch. This solution developed during the CNES study "Architectures d'antennes multifaisceaux pour constellations" proved to have equivalent radiating performance as conventional array of horn antennas but with the benefit of compactness. ${ }^{11}$

This antenna may support a bent pipe payload architecture with a fixed frequency plan in a single feed per beam configuration (SFPB). SFPB implies that each beam is amplified by a single high power amplifier (HPA) and requires constraints on amplification that are described further in Section 5.3. An issue with isoflux coverage is the beam lattice which is not regular, such as seen in traditional GEO hexagonal four-color schemes. ${ }^{12}$ A graph coloring algorithm based on DSATUR algorithm ${ }^{13}$ was implemented to allocate the frequency bands to each beam. This algorithm is efficient to provide frequency layouts avoiding the reuse of frequency bands between two neighbor beams that would generate interference. A six-color reuse scheme is utilized to reach enough spectral efficiency without exceeding the available power of the LEO satellite platform. Splitting the frequency band in more sub-bands would limit interference, at the cost of less bandwidth per beam.

The bent-pipe architecture in Figure 5A relies on traveling wave tubes amplifiers (TWTAs), chosen because of the power required and of the operating band (Ka band). OMUX are represented, but they do not play any role in the benchmark analysis. This architecture does not have DTP as it requires no routing on board, and a mapping between forward uplink and downlink frequencies can be implemented. This architecture delivers fixed coverage and fixed resource allocation, which may be optimal on uniform distributions (Figure $1 \mathrm{~A}$ ) but may not meet the demands of scenarios where the distribution of users in the FOV is not uniform (Figure 1B). More flexibility is in this case required, and other flexible solutions are proposed in the next sections.

\section{2 | Multiple beam coverage with beam hopping transponders}

As discussed in the Section 2, time domain solutions employing $\mathrm{BH}^{6}$ may offer flexibility for a payload with ferrite switches at post amplification level as illustrated in Figure 5B. In this case, as many ferrite switches as high power amplifiers are needed to allocate time slots. The integration of ferrite switches implies more mass, power, and accomodation requirements; consequently, this architecture is more complex than the previous one. Insertion losses due to the switches are neglected as they are estimated to be under $0.2 \mathrm{~dB} .^{7}$ As concerns the amplification architecture 
represented in Figure 5B, each TWTA is connected to a set of six possible beams, among which only one is illuminated during each time slot. In the architecture considered, 16 beams out of a total of 96 beams are active during each time slot. In each of these time slots, each beam illuminated emits the entire frequency band available for the forward downlink. The optimization algorithm for the time slot allocation to beams is presented in Section 5.3.

\section{3 | Multiple beam coverage with steering capabilities along ones axis}

The last architecture that is considered is a hybrid one. It utilizes a passive linear array of QOBF to form beams in columns of fixed coordinate $A$ and steers the beams along the $B$ axis thanks to an analog beam former (ABF), based on MMIC phase shifters. These beams are steered on a slot-by-slot basis. Flexibility is limited to steering beams along just one axis. However, this architecture is more flexible than the $\mathrm{BH}$ architecture because beams can be steered precisely over users positions, limiting losses in gain. This architecture also implements isoflux in one direction to compensate for the path loss previously described. QOBF losses are very low, ${ }^{9}$ so they are neglected for the analysis. For comparison purposes, time domain flexibility with $\mathrm{BH}$ is implemented also with this architecture. As with the previous architecture, 16 beams are illuminated per time slot but now with a maximum of two beams per column since each ABF is connected to two ports of the processor. Consequently, this payload cannot re-allocate power from one column to another. The HPA at the output of each ABF in Figure 6 corresponds to the distributed amplification along each steering column. For accommodation purposes and as the power required per HPA is lower than in SFPB architectures, solid state power amplifiers (SSPA) are chosen for this payload architecture.

\section{I PAYLOAD DESIGN}

Having defined the payload architectures to benchmark in Section 2, Section 3 proceeds with characterizing the components of the payloads. These include the radiation patterns of the antennas, how the antenna is monitored: $\mathrm{BH}$, beam steering and how amplification is distributed: SFPB, multiple feeds per beam.

\section{1 | Radiation patterns}

For the bent-pipe and the $\mathrm{BH}$ architectures, the radiation patterns of QOBF are estimated with a ray-tracing method. ${ }^{14}$ The radiation of the antenna subsystem (doubly curved reflector illuminated by QOBF) is then calculated with GRASP, a commercial software package for satellite antenna design and analysis. A 10- $\mathrm{dB}$ taper of the reflector ensures lower side lobes to mitigate interbeam interference and interference with GEO satellites.

For the steerable case, the simulated radiation patterns of the QOBF are combined in a phased array. Each column of beams represents one input port of a QOBF. Amplitude coefficients of the linear array are chosen according to a Taylor law to reduce the side lobe levels on the north-south axis. On the west-east axis, side lobes are mitigated thanks to the tapering of QOBF apertures.

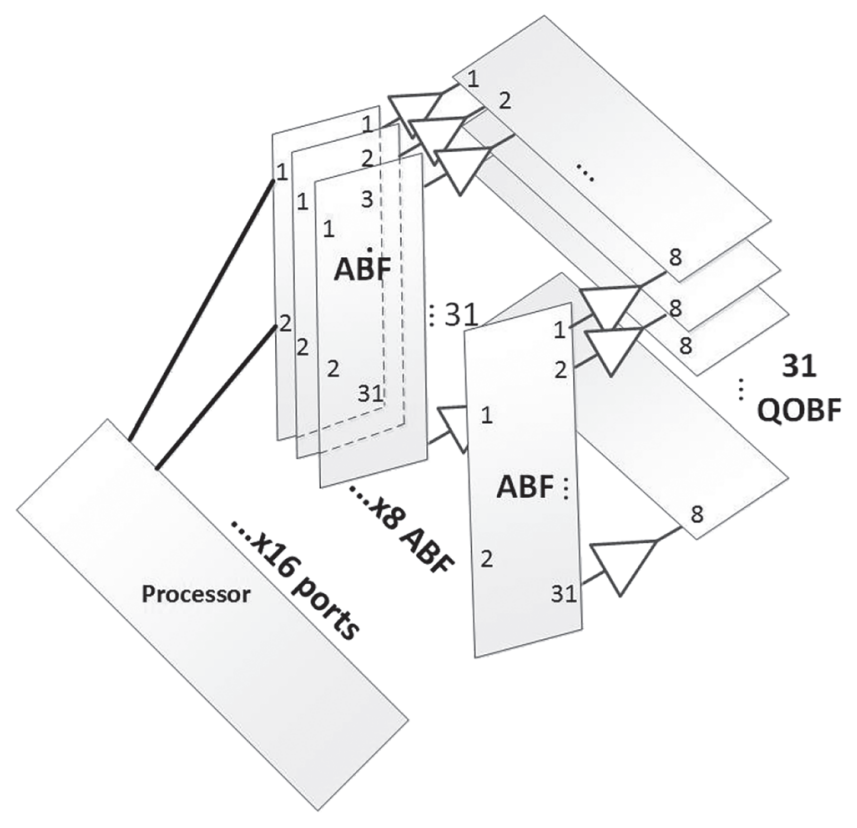

FIGURE 6 Hybrid steering architecture with analog plus quasi-optical beam forming 
FIGURE 7 Directive beams are generated over users with isoflux implemented in the A direction [Colour figure can be viewed at wileyonlinelibrary.com]

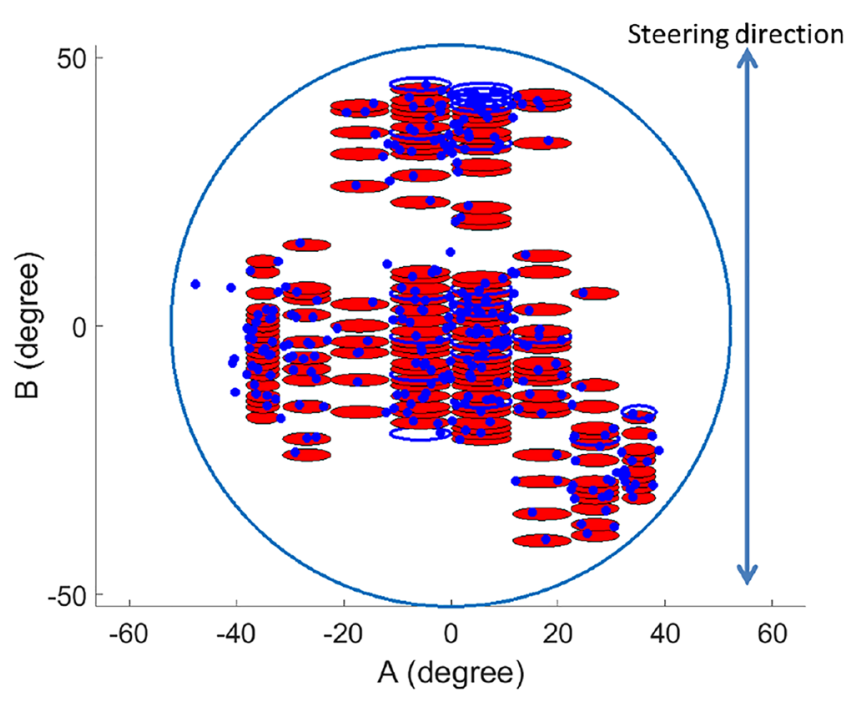

\section{2 | Antenna monitoring}

Two antenna monitoring techniques are taken into account: $\mathrm{BH}$ and beam steering. In $\mathrm{BH}$, beams are already formed and follow the bent-pipe architecture lattice. $\mathrm{BH}$ consists of allocating the optimal time slots to the beams in order to minimize interference under constraints relating to the amplifiers (each amplifier is shared among a fixed set of beams) and the time slots. The hybrid architecture relies on beam steering. In this case, the antenna is able to steer toward the optimal directions that are not predefined as in $\mathrm{BH}$. To reduce the complexity of the optimization in the beam steering cases, the FOV of the antenna is sampled with possible beam positions as illustrated in Figure 7. Subsequently, beams that serve users are kept and considered in the resource allocation problem to reduce the size of the optimization problem.

\section{3 | Amplifiers}

One of the constraints of the resource allocation algorithm is related to power sharing among beams (constraint later specified in Section 5.3). Power sharing is formalized in binary matrices $M$ of size $N_{\text {HPA }} \times N_{b}$, with $N_{\text {HPA }}$ the number of HPAs which can be either TWTAs or banks of distributed SSPAs, and $N_{b}$ the number of beams. $M_{h, b}$ equals 1 when power from HPA $h$ can be allocated to beam $b, 0$ otherwise. For mathematical notation clarity, matrices are represented in bold below. In the BH architecture, the connection matrix links HPAs to the beams they can potentially illuminate. The connection matrix of the bent-pipe and the $\mathrm{BH}$ architectures is defined in Equation (3).

$$
\left.M=\left[\begin{array}{ccccccccc}
6 \text { beams per amplifiers } & \multicolumn{1}{c}{1} & 1 & 1 & 1 & 1 & 0 & \cdots & 0 \\
0 & 0 & 0 & 0 & 0 & 0 & 1 & \cdots & 0 \\
\cdots & \cdots & \cdots & \cdots & \cdots & \cdots & \cdots & \ddots & \cdots \\
0 & 0 & 0 & 0 & 0 & 0 & 0 & \cdots & 1
\end{array}\right]\right\} 16 \text { amplifiers }
$$

For the steering architecture, each steering column has a distributed amplification: In each column of beams as represented in Figure 7, beams share a common bank of power formed by the amplifiers at the output of each ABF (see Figure 6). It is to be noted that for this architecture, the matrix depends on the position of the preformed beams, which are deduced from the position of users in the FOV as illustrated in Figure 7.

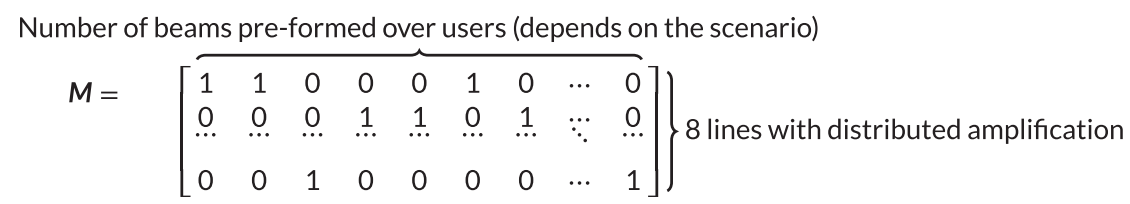

The connection matrix was introduced in Cocco et $\mathrm{al}^{15}$ to express the constraints for a resource allocation problem in multibeam configurations. Power constraints are further detailed in Section 5.3.

\section{4 | RESOURCE ALLOCATION}

\subsection{Generation of traffic scenarios}

As a minimum, an objective of $100 \mathrm{Mbps}$ per household ${ }^{16}$ was set as a target by the European Commission. This value is considered to be a realistic data rate demand per terminal. Scenarios of user distributions were generated by spreading differently 300 user terminals with aforementioned 


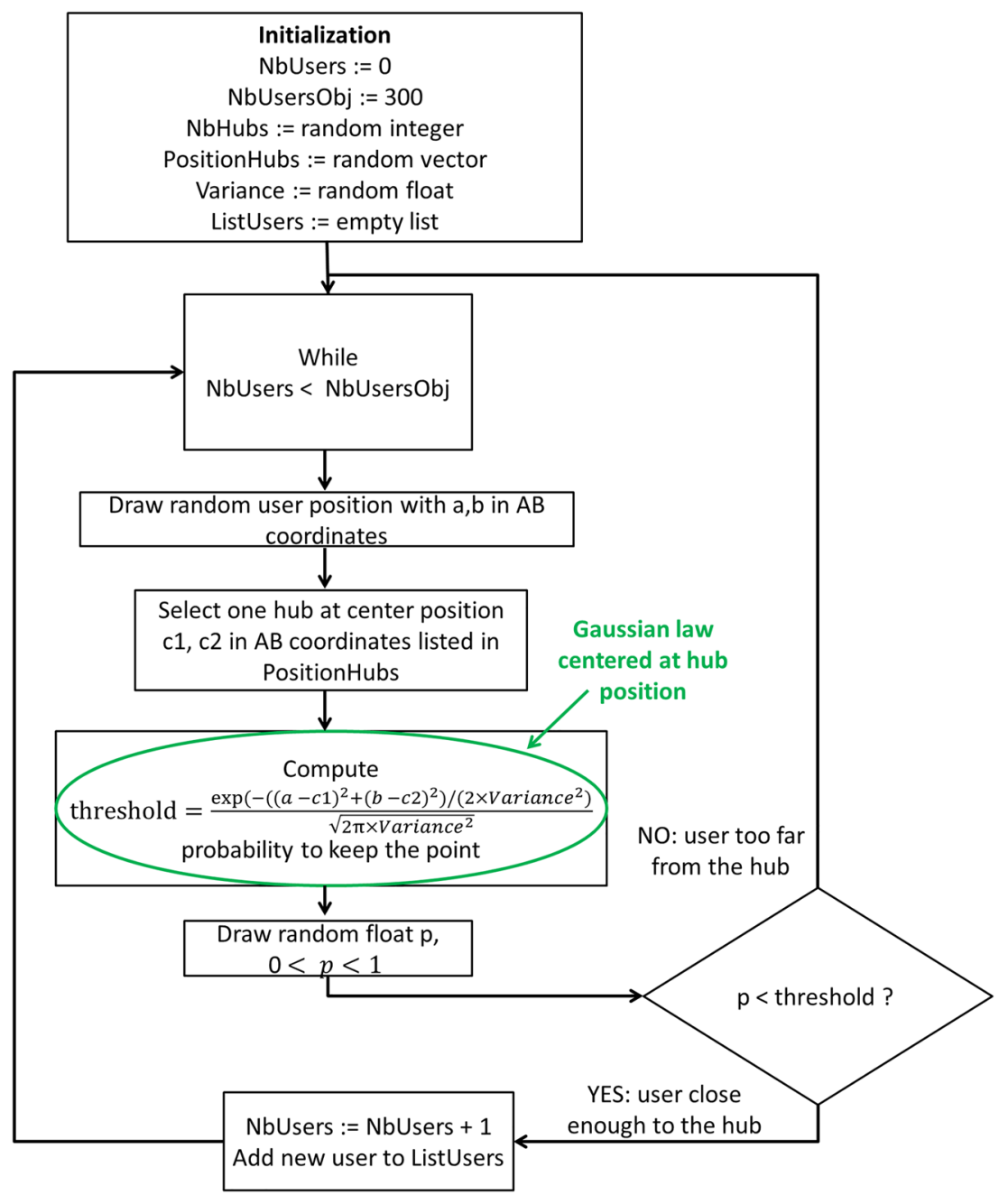

FIGURE 8 User distributions generation process [Colour figure can be viewed at wileyonlinelibrary.com]

traffic demands. In this way, the target throughput per satellite would be $30 \mathrm{Gbps}$, which is in the range of current megaconstellation projects. ${ }^{5}$ For the purposes of link budget computations, all user antennas are assumed to be $60 \mathrm{~cm}$ in diameter, which corresponds to a fixed satellite service application with a figure of merit $\mathrm{G} / T=15 \mathrm{~dB} / \mathrm{K}$. A method is proposed to generate user distributions that would cover all statistically possible profiles of distributions from uniform distribution to uneven ones. To generate a uniform scenario, $A B$ coordinate pairs in the antenna FOV are randomly selected following a uniform law. To add non-uniformity, denser user demands must be generated in some areas. These areas of higher traffic density, in the remaining referred to as hubs, are centered around positions that are randomly chosen across the FOV. Users are drawn according to the uniform law and randomly kept following a Gaussian law centered at hubs positions. The variance of this law and the number of hubs can be tuned to increase the non-uniformity of the distribution. The process of scenario generation is described in Figure 8 .

\subsection{Distribution characterization of traffic scenarios}

Here, we define a parameter that captures in a single number how uniformly user traffic is spread across the satellite FOV. We start by producing the user demand distribution over the coverage area. This is performed by partitioning the FOV into elliptical cells each of surface area commensurate to the dimension of the average beam footprint on Earth. An example of cell lattice is given in Figure 9A. Note that the number of lattice cells is not related with the actual number of beams generated by the payload. Based on a known traffic scenario, we can then calculate the aggregate traffic demand, ATD, per cell. The parameter to quantify non-uniformity, denoted $\mu$, is then defined in Equation (5) as the standard deviation of the aggregated demands in each cell normalized by the mean of this parameter in these cells.

$$
\mu=\frac{\text { standard deviation of ATD in all the cells in the coverage }}{\text { mean of ATD in all the cells over coverage }}
$$

Following the approach proposed in this paper, ${ }^{17}$ instead of ATD in Equation (5), we can use a weighted aggregate traffic demand, WATD, per cell such that traffic closer to the center of the cell has a higher contribution to the aggregated value. The expression for the weighted aggregate traffic demand for cell $c$ with center coordinates $\left(c_{a}, c_{b}\right)$ follows Equation (6). 
FIGURE 9 A, Elliptical iso-Earth projection surface cells to compute WATD; B, WATD overlapped with users positions represented by dots [Colour figure can be viewed at wileyonlinelibrary.com]
(A)

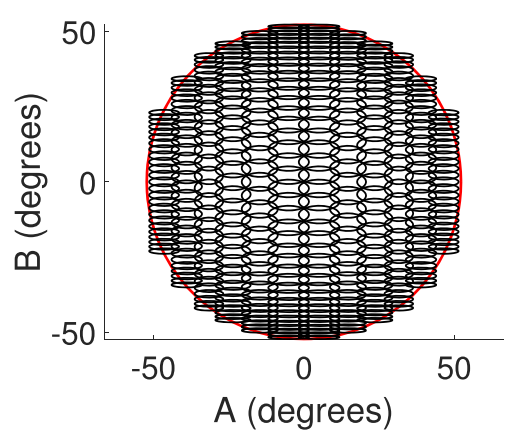

(B)

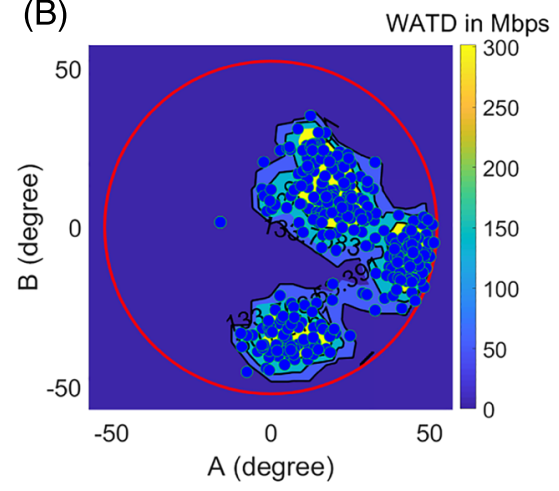

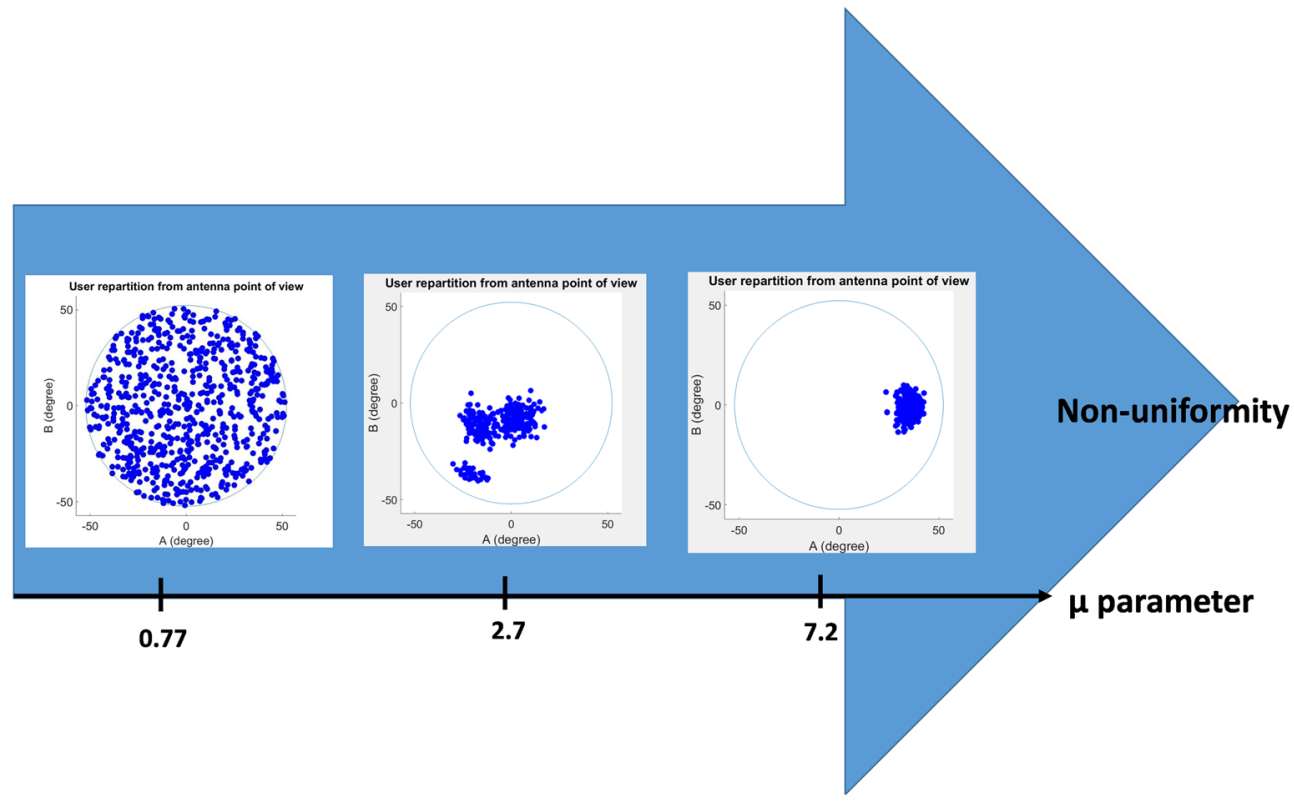
distribution with non-uniformity parameter [Colour figure can be viewed at wileyonlinelibrary.com]

$$
\operatorname{WATD}(c)=\sum_{s \in D_{c}} T(s)\left(1-\sqrt{\frac{4\left(s_{a}-c_{a}\right)^{2}}{\omega_{a}^{2}}+\frac{4\left(s_{b}-c_{b}\right)^{2}}{\omega_{b}^{2}}}\right)
$$

In Equation (6), $\omega_{a}$ and $\omega_{b}$ are the major and minor axes of the elliptical cell while the user terminal has coordinates $\left(s_{a}, s_{b}\right)$. $T(s)$ is the demand for user $s, D_{c}$, the set of users within the cell $c$. For each cell, ATD is increasing with the aggregated demand of the user terminals in the cell as depicted in Figure 9B. WATD is further weighted by the distance of users from the center of the cell to account for the distribution of users within the cell, such that for a user at the edge of an elliptical cell the contribution to WATD converges to 0 . Conversely, if users that raise a fixed ATD in a cell are packed nearer its center, WATD would be higher and converge toward ATD.

Based on the above definitions, Figure 10 depicts examples of user distributions and the calculated value of the non-uniformity coefficient $\mu$. In Figure 10 and for the rest of the paper, we have used WATD in the ratio of Equation (6). The results in Section 6 exhibit the link between the non-uniformity parameter and the ability of the payload architectures to achieve high throughputs. High WATD limits the throughputs allocated because of the limited frequency band available and the limits to reuse this resource with wide beams due to inter beam interference.

\subsection{Description of the resource allocation algorithm}

In the case of the $\mathrm{BH}$ and steerable payload architectures, a resource allocation algorithm is needed to efficiently allocate time slots to beams and concentrate the time slots over the busy regions while avoiding interference. A simulated annealing (SA) algorithm is used to solve this NP-hard problem that is close to the frequency assignment problem. ${ }^{18}$ SA brings advantageous features in terms of its simplicity and convergence; studies involving a given traffic scenario with different starting points have shown to converge to the same optimum solution, thereby providing confidence on the accuracy of the method. This type of algorithm was also used in Cocco et al ${ }^{15}$ to solve the resource allocation problem and is described by a flow graph in Figure 11.

This algorithm provides the optimum time slots to beams association by allocating time slots to beams with unserved demand taking into account interference. An initial solution is provided to the algorithm, then the algorithm explores the domain of feasible solutions with the capacity of new random solutions being estimated. During this exploration phase, the algorithm is more likely to accept solutions with poor 


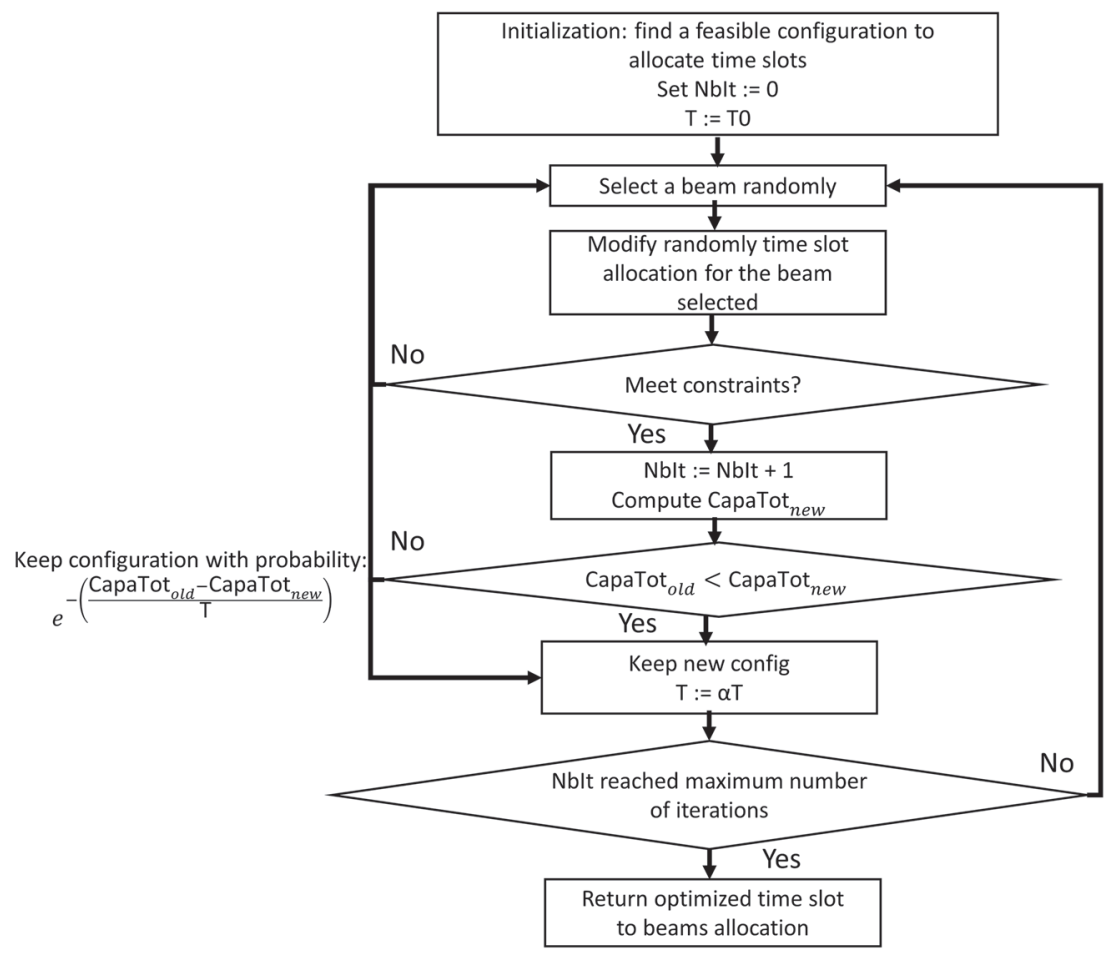

FIGURE 11 Simulated annealing algorithm [Colour figure can be viewed at wileyonlinelibrary.com]

\begin{tabular}{|ll|}
\hline Initial temperature & $1000 \mathrm{~K}$ \\
\hline Cooling factor $\alpha$ & 0.9993 \\
\hline Number of iterations & 20000 \\
\hline
\end{tabular}

TABLE 1 Simulated annealing algorithm parameters

throughputs. This probability of acceptance decreases with the number of iterations as the temperature $T$ decreases thanks to the cooling factor $\alpha$. In the end, the algorithm only accepts the modifications in the time slots allocation that improve the current configuration in a local research. The parameters determined for the SA algorithm are summarized in Table 1.

The initial temperature was selected to allow a wide range of exploration at the beginning of the iterations. The cooling factor and the number of iterations are jointly chosen to balance exploration and local research phases.

The allocation of a time slot to a beam is stored in the binary matrix $T, T \in \mathcal{T} \subset \mathbb{N}^{N_{b} \times N_{t}}$ where $T_{b, l}$ equals 0 if the beam $b$ is off at time slot $I$ and 1 if this beam is on during this time slot. $\mathcal{T}$ is the set of time slot matrices that adhere to the following payload constraints:

- For all architectures, power constraints must be met: It is impossible to allocate more power to a set of beams than the amount produced by the HPA connected to these beams by the $M$ matrix defined in Section 4.3. This corresponds to the condition $M\left(T_{1} \odot W\right) \leq P$, where $T_{1}$ is the Ith column of matrix $T$ and $\odot$ the Hadamard product that multiplies element wise vectors and matrices. The vector $P, P \in \mathbb{R}^{N_{H P A}} \times 1$ contains the maximum power that can be allocated to beams for each amplifier or distributed amplification. $N_{\text {HPA }}$ is the number of HPAs in the bent-pipe and $\mathrm{BH}$ architectures or the number of steering columns which corresponds to $\mathrm{N}_{\text {HPA }}$ distributed amplification banks. The vector $W, W \in \mathbb{R}^{N_{b} \times 1}$ stores the power allocated per beam.

- A payload constraint that applies to all payload architectures implementing $\mathrm{BH}$ is the number of beams illuminated at each time slot that here is assumed to be 16 , which can be formalized by for all time slots $l, \sum_{b} T_{b, l} \leq 16$.

- In the case of SFPB architectures, the beam to amplifier connection matrix must be taken into account and must ensure that an amplifier does not amplify more than one beam within the same frequency band. This constraint is not taken into account in this optimization because at each time slot, each amplifier illuminates at most one beam over the entire frequency band available. However, in the case of a system with flexible frequency slot allocation, this constraint must be accounted for.

- For the hybrid architecture, due to beam forming constraints in the DTP, only two beams at maximum per column of fixed coordinate A can be formed. This constraint can be formalized for all time slot $I$ and for all steering column $C_{i}$ with $i$ ranging from 1 to 8 , $\sum_{b \in C_{i}} T_{b, l} \leq 2$.

To determine the data rate that can be reliably transmitted to each user, the spectral efficiencies that can be achieved are calculated using carrier-to-noise power ratios and interference levels and considering the DVB-S2 standard. A design choice to have a constant power density (units $\mathrm{W} / \mathrm{Hz}$ ) per beam is followed to have comparable spectral efficiencies over the coverage. Combined with the isoflux requirements presented 
in Section 3.1, this policy ensures an iso-EIRP density over the coverage area to achieve a quasi-uniform spectral efficiency. Users are allocated to beams from which centers they are the closest. In Equation (7), $(C / N)_{i}^{j}$ represents the carrier-to-noise ratio of user $j$ belonging to beam $i$

$$
\left(\frac{C}{N}\right)_{i}^{j}=P_{i} g_{i}^{j}\left(\frac{4 \pi d_{j}}{\lambda}\right)^{-2}\left(\frac{G}{T}\right)_{j} \frac{1}{k B_{i}},
$$

where $P_{i}$ is the power allocated to beam $i, g_{i}^{j}$ the gain of beam $i$ toward user $j, d_{j}$ the distance between the satellite and user $j, k$ the Boltzmann constant, $B_{i}$ the frequency bandwidth allocated to beam $i$, and $(G / T)_{j}$ the antenna gain-to-noise-temperature of the user terminal.

The carrier to interference power ratio of user $j$ belonging to beam $i$ is computed in the following way:

$$
\left(\frac{C}{l}\right)_{i}^{j}=\frac{P_{i} g_{i}^{j}}{\sum_{i^{\prime}, i^{\prime} \neq i} P_{i^{\prime}} g_{i^{\prime}}^{j} b_{i^{\prime}, i}},
$$

where $b_{i^{\prime}, i}$ is the overlapping time ratio between beams $i^{\prime}$ and $i$. If these two beams are simultaneously illuminated during all the time slots they are active, this coefficient equals 1 . If they are never illuminated at the same time, this coefficient equals 0 .

For user resource access, a TDMA scheme with one carrier per beam is assumed. When a beam is allocated a certain time slot, the frequency band available to users is allocated in a greedy way such that users with best link budget are served first. Spectral efficiencies are computed according to DVB-S2 standard and SINR levels given in Equation (9) utilizing Adaptive Coding and Modulation (ACM) technics. ACM helps to maintain a communication link between the satellite and user terminals with lower SINR signals at the cost of lower spectral efficiencies. On the opposite, higher spectral efficiencies can be reached in the case of high SINR with the use of complex symbol constellations. $(C / I)_{\text {intermod }}$ are estimated from the modulation and output back-off operated at amplifiers level.Aloisio et al. ${ }^{19}$ and D'Addio and Valenta ${ }^{20}$ provide values of intermodulation interference respectively for TWTA and SSPA.

$$
\operatorname{SINR}_{i}^{j}=\frac{1}{\left(\left(\frac{c}{N}\right)_{i}^{j}\right)^{-1}+\left(\left(\frac{c}{l}\right)_{i}^{j}\right)^{-1}+\left(\left(\frac{c}{l_{\text {intermod }}}\right)_{i}^{j}\right)^{-1}}
$$

In the case of the steering architecture, beams are generated at user positions to simplify the resource allocation problem (see Figure 7). Radiation pattern models are described in Section 4.2).

\section{5 | RESULTS}

\section{1 | Architectures performance}

The total available bandwidth for the forward downlink is assumed to be $2.5 \mathrm{GHz}$. A total of 50 different traffic scenarios with varying aggregated demands are generated following the methodology presented in Section 5.1. All the payloads were designed to provide the same throughput on a uniform scenario with an aggregated demand of $30 \mathrm{Gbps}$, which is the maximum throughput targeted per satellite of the megaconstellation.

Figure 12 presents how each payload architecture performs on the scenarios generated with in all cases 30 Gbps of aggregated demand (only the geographical distribution of this demand varies). The matching ratio (MR) is defined as the ratio between the total throughput allocated by the payload over the aggregated user demand in the FOV of the satellite. For all the payload architectures, MR tends to decrease with non-uniformity. For the bent-pipe architecture, the non-flexibility of the resource allocation limits quickly the matching ratios achieved. BH achieves better throughputs, reaching more than two times the throughput of the bent-pipe architecture for $\mu$ over 2 . The hybrid architecture performs the best. Its benefits are particularly observable for $\mu$ between 1 and 7. For $\mu$ values over 7, corresponding to high traffic non-uniformity, the performance of the steering architecture converges toward that of $\mathrm{BH}$. Payloads architectures with different levels of complexity demonstrate different levels of flexibility. The most flexible solution, the beam steering architecture, proved to have the best results in matching ratio over the highest range of $\mu$ parameters. However, it is also the most complex architecture as it will be further discussed in Section 5.3. The bent-pipe architecture is the least flexible with inferior matching ratios for most $\mu$ parameters, especially for the highest $\mu$, but it is the most simple. $\mathrm{BH}$ is an intermediate solution between these two architectures in terms of flexibility and complexity. The same conclusions can be drawn from scenarios with total aggregated demand of 10 (Figure 12A) and $20 \mathrm{Gbps}$ (Figure 12B) with better MR achieved as the aggregated demands are lower in these scenarios. These trends have been confirmed for a scenario including 1000 users and a total demand of $20 \mathrm{Gbps}$ (not shown here for brevity).

The $\mu$ parameter characterizes the different levels of non-uniformity over which a user distribution can range. However, all $\mu$ values may not be reached in realistic scenarios and possibly might occur with different probabilities. In the next section, an analysis employs the non-uniformity analysis results to select the optimal payload architectures for specific worldwide user distributions. 


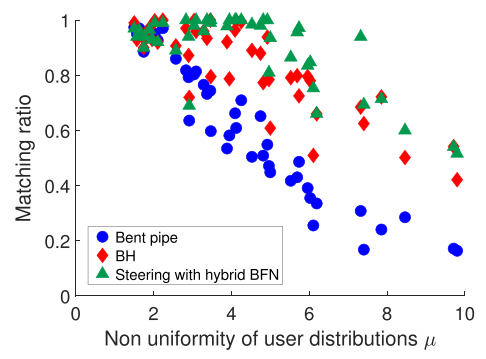

(A) Total demand of $10 \mathrm{Gbps}$

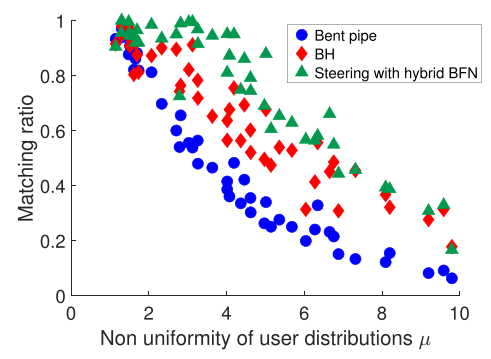

(B) Total demand of $20 \mathrm{Gbps}$

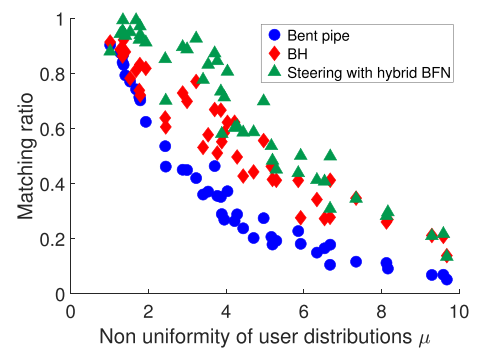

(C) Total demand of $30 \mathrm{Gbps}$

FIGURE 12 Variation of matching ratio achieved by the payload architectures with non-uniformity $\mathrm{A}$, total demand of $10 \mathrm{Gbps}$; B, total demand of $20 \mathrm{Gbps}$; C, total demand of $30 \mathrm{Gbps}$ [Colour figure can be viewed at wileyonlinelibrary.com]

\subsection{Analysis on an orbit with a realistic traffic}

In the actual case of a LEO satellite, estimating the throughput at each orbital position is a costly process as the SA algorithm should be run over one or more complete orbits for each payload architecture considered. An analytical approach is proposed in this section to quickly benchmark payload architectures for specific traffic scenarios. A simple model of MR, as a function of the aggregated demand and non-uniformity, is proposed. A statistical overall capacity achieved by each architecture over two polar orbits is deduced from the models and permits one to determine the best architecture for two different realistic scenarios of users' worldwide distributions. Polar orbits were chosen to achieve global coverage of Earth. These types of orbits are planned to be used by the OneWeb and Telesat megaconstellations. ${ }^{5}$

MR functions are modeled with one parameter $\mu_{0}$ defined in Equation (10). This function is chosen because as $\mu$ approaches 0 (uniformity), the MR converges to 1 (all the user demand is met). $\mu$ was squared to reflect the fact that above a certain non-uniformity, MR decreases more slowly. As $\mu_{0}$ grows, the MR decreases more rapidly.

$$
M R_{\text {model }}(\mu)=1-\exp \left(\frac{-\mu_{0}}{\mu^{2}}\right)
$$

$\mu_{0}$ is higher for the steering architecture, showing its greater flexibility compared with the other solutions (see Figure 13 for the results at $30 \mathrm{Gbps}$; the results for other values of aggregated demands are included in Annex I, Figure A1 and Figure A2). BH is the second architecture with higher $\mu_{0}$ and the least flexible bent-pipe architecture has the lowest $\mu_{0}$ parameter.

$\mu_{0}$ values are computed with the least square method, which consists of minimizing the sum of the squares of the residuals $r_{i}=M R_{i}-M R_{\text {model }} \quad\left(\mu_{i}\right)$ , with, $i=1, \ldots n$, where $n$ is the number of user distribution scenarios, here $50 ; \mu_{i}$ are the non-uniformities of each user distributions generated, computed with the method described in Section 5.2. $M R_{i}$ is the associated matching ratio computed with SA and $M R_{\text {model }}$ the function expressed in Equation (10).

A payload benchmark study based on the non-uniformity of realistic traffic issued from a commercial forecast analysis is next presented. These data are derived from the ESA study Next Generation High Data Rate Trunking Systems ${ }^{21}$ in the framework of an ARTES Future Program. The first traffic profile contains a mix of data for aeronautical, maritime, and terrestrial markets exhibited in Figure 14A. The other traffic profile is only based on the aeronautical data of this study (see Figure 14B). The scenarios are adapted such that the maximum demand in the satellite FOV never exceeds 30 Gbps throughput for which the payloads were initially designed.

In Figure 15, the mixed demand presented in Figure 14A seen by the satellite orbiting at $1200 \mathrm{~km}$ is plotted with the non-uniformity of the traffic in its FOV and the throughput allocated by different payloads. The results for the aero case displayed in Figure 14B are presented in Annex II, Figure B1.

In the cases where the aggregated demand is less than $10 \mathrm{Gbps}$, it is assumed that the satellite completely fulfills the demand. Otherwise, for each payload architecture and for the demand at each orbital position, the parameter $\mu_{0}$ introduced in Equation (10) is linearly interpolated from the $\mu_{0}$ obtained for aggregated demands of 10,20, and $30 \mathrm{Gbps}$. The gaps in the curves correspond to the flyovers of the South pole where there is no demand. Non-uniformity may reach high values; however, most of the time, these values are associated with low aggregated demands so payloads architectures are able to meet the demand in these cases. 
FIGURE 13 Least square error

interpolation results with the matching ratio function model selected for $A$, the bent-pipe; $B$, beam hopping; and $C$, beam steering architectures for a total demand per scenario of $30 \mathrm{Gbps}$ [Colour figure can be viewed at wileyonlinelibrary.com]

FIGURE 14 Demand geographical distribution. A, Mix of traffic scenario (aero, maritime, terrestrial); B, aero traffic scenario [Colour figure can be viewed at wileyonlinelibrary.com]

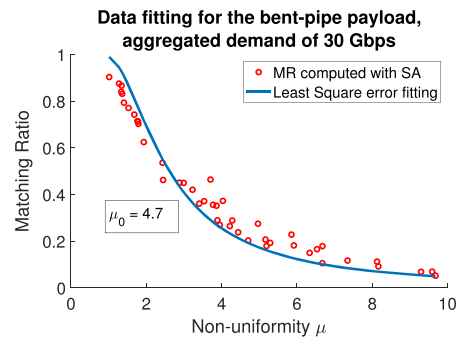

(A) Bent-pipe

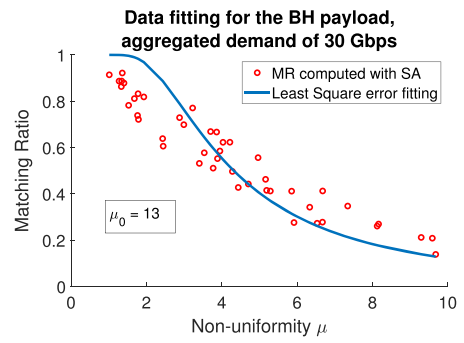

(B) Beam Hopping

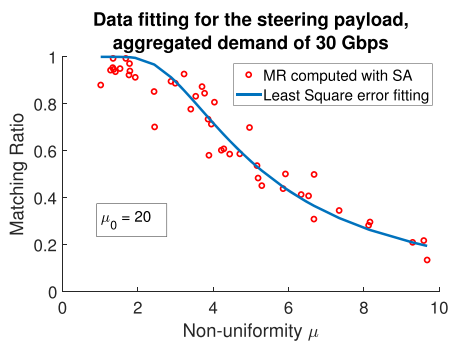

(C) Beam Steering

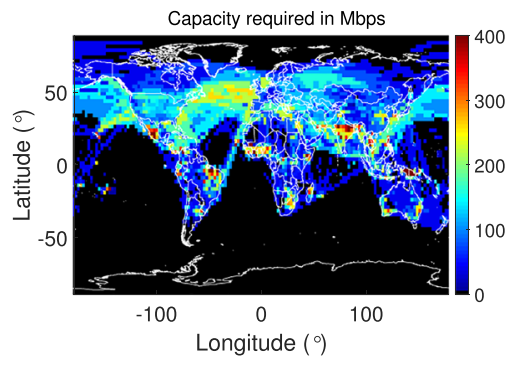

(A) Mix of traffic scenario (aero, maritime, terrestrial

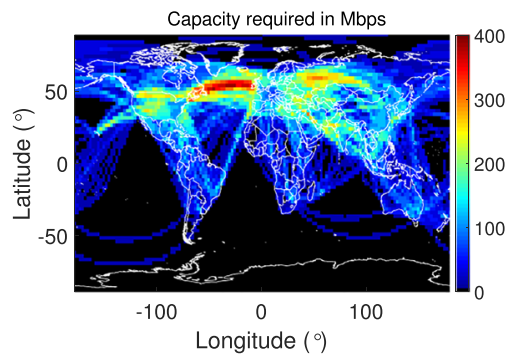

(B) Aero traffic scenario

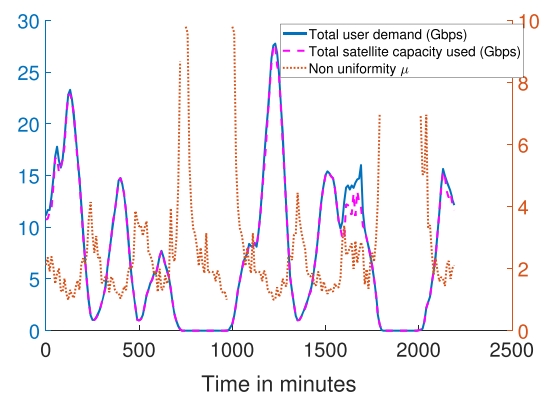

(A) Bent-pipe

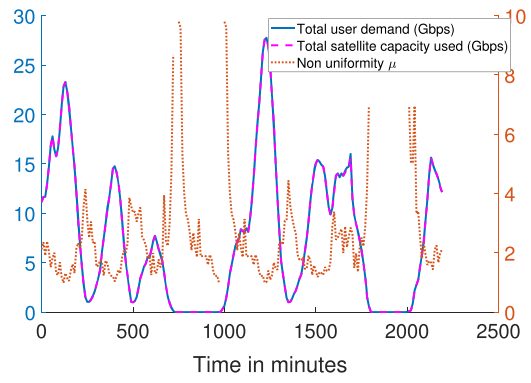

(B) Beam Hopping
FIGURE 15 For the mixed traffic scenario, variation of non-uniformity, total user demand, and estimated capacity used over two 110-minute orbits for the A, bent-pipe; $B$, bea,m hopping; and $\mathrm{C}$, steering architectures [Colour figure can be viewed at wileyonlinelibrary.com]

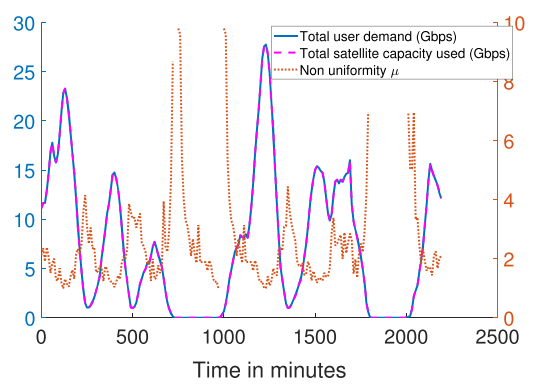

(C) Beam Steering 
TABLE 2 Payloads performances derived from the model

\begin{tabular}{|c|c|c|c|c|}
\hline \multirow[t]{2}{*}{ Architectures } & \multicolumn{2}{|l|}{ Mixed traffic scenario } & \multicolumn{2}{|l|}{ Aero traffic scenario } \\
\hline & Average throughput over orbits (Gbps) & Minimum MR & Average throughputs over orbits (Gbps) & Minimum MR \\
\hline Bent-pipe & 7.2 & 0.71 & 5.7 & 0.89 \\
\hline $\mathrm{BH}$ & 7.4 & 0.97 & 5.8 & 0.99 \\
\hline Steering & 7.4 & 0.99 & 5.8 & 0.99 \\
\hline
\end{tabular}

\begin{tabular}{llll} 
& Bent-pipe & Beam hopping & Beam steering \\
\hline Mass ferrite switches & 0 & $8.8 \mathrm{~kg}$ & 0 \\
\hline Mass ABFs & 0 & 0 & $3 \mathrm{~kg}$ \\
\hline Mass QOBFs & $19 \mathrm{~kg}$ & $19 \mathrm{~kg}$ & $49 \mathrm{~kg}$ \\
\hline Total mass estimated & $19 \mathrm{~kg}$ & $28 \mathrm{~kg}$ & $52 \mathrm{~kg}$ \\
\hline DC power consumed by amplifiers & $830 \mathrm{~W}$ & $830 \mathrm{~W}$ & $1400 \mathrm{~W}$ \\
\hline
\end{tabular}

TABLE 3 Mass and power budgets

For the mixed scenarios, in the $\mathrm{BH}$ and in the steering cases, the throughputs follow the demand with MR near 1 along all the orbits. In Figure 15A, the MR of the bent-pipe architecture decreases to 0.7 at the 1600th minute when the satellite flies over New Guinea where the backhaul and inland terrestrial demands are expected to be important. The high demands in these sparse islands surrounded by the Pacific ocean induce highly uneven user distributions. The results for the aero distribution are put in Annex II as in all cases the throughputs follow closely the demand so no particular interpretation can be made with these curves.

The average throughput and the minimum MR over the orbital positions are shown in Table 2 for both traffic scenarios. The mixed scenario proved to have less uniform user distributions than the aero scenario due to the scattered demand in islands and land terminals that require more data rate than aero terminals. The New Guinea case cited above illustrates this rise of demand non-uniformity. The choice of the optimum payload may be driven by the maximum average throughput provided. $\mathrm{BH}$ would be chosen in this case as it performs similarly to the steering architecture but with less complexity in the payload. Another driver may be the highest minimum MR over the coverage. In this case, the steering architecture is the best one with $99 \%$ of the total demand matched for all orbital positions. However, the choice for the payload architecture must also account for the complexity of each candidate design. The next subsection deals with an approach to determine the most appropriate payload given the results presented previously and the complexity of each payload architecture.

\section{3 | Trade-off between satellite used capacity and payload complexity}

The previous subsections (5.1 and 5.2) compared the different payload architectures based on the capacity they could effectively achieve. In subsection 5.2, the results obtained from realistic scenarios present the benefits of $\mathrm{BH}$ and beam steering compared with the bent-pipe architecture. These benefits were demonstrated in terms of average throughput and minimum MR over two 110 minutes orbits. To conclude the benchmark, this subsection examines the complexity of such flexible payload architectures. At first, the estimation of the complexity focuses on the payload mass and DC power consumption. On the one hand, mass is related with accommodation constraints and launch costs. On the other hand, power is linked with constraints on thermal design for heat dissipation as well as the mass and cost of HPAs, solar panels, and batteries. Mass and power therefore play a determinant role in the assessment of the satellite complexity.

The estimation of mass takes into account the QOBFs (which have the role of beam formers and radiating elements) and reflectors for the bent-pipe payload. In Tubau et al, ${ }^{11}$ figures for the mass of the QOBFs are given as a function of the number of beams formed. According to this reference, the configuration QOBFs plus reflectors entails 2.5 beams $/ \mathrm{kg}$. With recent developments mentioned in Tubau et al ${ }^{11}$ on QOBF with up to $54^{\circ}$ scanning angle and new manufacturing techniques (including molded plastics and additive manufacturing), the mass can be reduced to achieve 5.1 beams $/ \mathrm{kg}$. For the $\mathrm{BH}$ architecture, ferrite switches are additionally taken into account. Commercial solutions are available, and the estimated mass is $550 \mathrm{~g}$ per ferrite switch. For the beam steering architecture, the analog beam former mass was estimated to $3 \mathrm{~kg}$. Mass estimations are detailed below in Table 3.

To a first approximation, the power consumed only accounts for the HPAs as they are expected to play a dominant role in power consumption. Their efficiency depends on the amplifier technology: SSPAs have 35\% of estimated power efficiency, whereas TWTAs have an estimated 60\% power efficiency. All architectures have a total of $500 \mathrm{~W}$ aggregated RF power. Even if these mass and power budgets are not exhaustive, they give an overview of the complexity of the payload subsystems that are benchmarked with the main components that contribute to the mass and power budgets.

Considering the earlier results and Table 3, BH seems to offer a preferential trade-off with average throughputs and minimum MR reaching closely the ones of the beam steering architecture in mixed and aeronautical scenarios. These performances are leveraged with 1.9 times less mass and $40 \%$ less power consumption than the beam steering architecture. Even if the bent-pipe payload is the less complex architecture with $30 \%$ less mass than $\mathrm{BH}$, its lowest MR (70\%) does not make it a reliable solution in the scenarios analyzed. In use cases with narrower FOVs and less expected demand, this configuration, analogous to the OneWeb approach, could have been considered.

The diagram in Figure 16 sums up the methodology presented in the paper. 


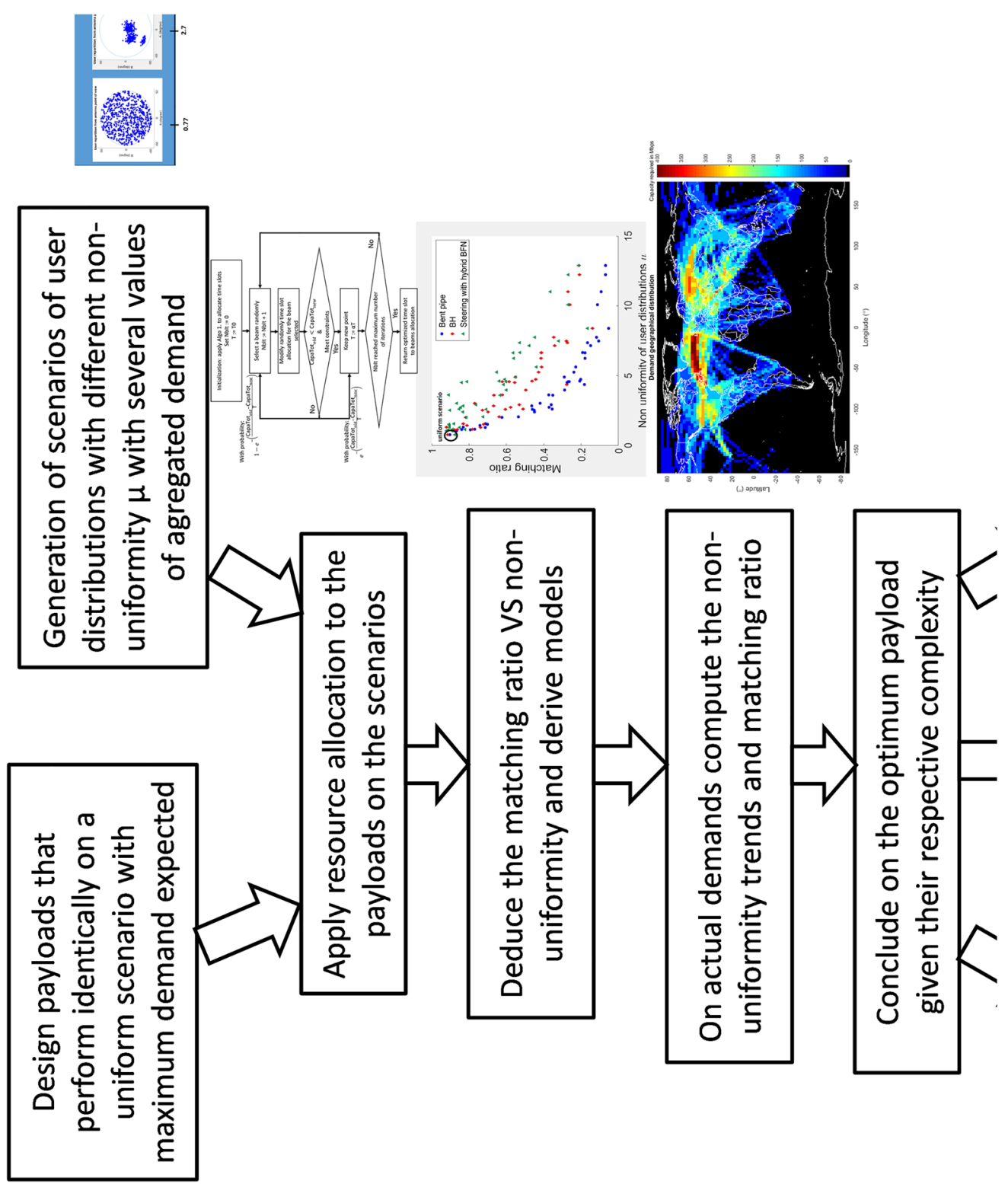




\section{6 | CONCLUSION}

A method was developed to benchmark the flexibility of payloads and antenna subsystems to meet the aggregate demands of uneven traffic distributions. It has been applied to several multiple beam antenna architectures in a megaconstellation use case. In this use case, the trade-off between complexity and flexibility is crucial. Orbiting satellites experience a high diversity of user distributions, but at the same time, low-cost systems are needed to limit the overall cost of launching a megaconstellation. To benchmark the presented solutions, a method to compare the flexibility of antenna and payload solutions in servicing non-uniform user demand distributions was developed. The study considered three payload architectures all involving an innovative quasi-optical beamformer as a primary radiator. The three architectures enable beam hopping, beam steering, as well as static beam allocation, respectively. A resource allocation algorithm was coded to estimate the performance of each payload in each scenario. A measure of the non-uniformity of the user distribution was introduced to observe how non-uniformity affects the throughput of each solution. The choice for the more appropriate payload depends on the non-uniformity parameter and demands encountered during the satellite orbits and the complexity of each payload architecture. Complexities have in turn been estimated in regard to the mass and power consumed by each payload. The beam hopping and the beam steering architectures prove to be efficient solutions compared with the bent-pipe architecture to adapt flexibly to the non-uniformity of realistic demands over LEO orbits. However, with 1.9 times less mass and $40 \%$ less power consumed, the beam hopping architecture appears to be the optimal payload design.

\section{ACKNOWLEDGMENTS}

The quasi-optical beamformers of the bent-pipe and $\mathrm{BH}$ architectures were designed in the context of the CNES study "Architectures d'antennes multifaisceaux pour constellations". This work was supported by the European Commission under H2020 project REVOLVE (MSCA-ITN-2016-722840)

\section{ORCID}

Florian Vidal (D) https://orcid.org/0000-0001-6834-1475

\section{REFERENCES}

1. Evans B, Werner M, Lutz E, et al. Integration of satellite and terrestrial systems in future multimedia communications. IEEE Wirel Commun. 2005;12(5):72-80.

2. Maral G, Bousquet M. Satellite communications systems: Wiley; 2005.

3. Palacin B, Fonseca NJG, Romier M, et al. Multibeam antennas for very high throughput satellites in europe: technologies and trends. In: 2017 11th European Conference on Antennas and Propagation (EUCAP); 2017:2413-2417.

4. Agiwal M., Roy A, Saxena N. Next generation 5G wireless networks: a comprehensive survey. IEEE Commun Surv Tutor. 2016;18(18):1617-1655.

5. del Portillo I, Cameron BG, Crawley EF. A technical comparison of three low earth orbit satellite constellation systems to provide global broadband. 69th International Astronautical Congress (IAC). 2018.

6. Anzalchi J, et al. Beam hopping in multi-beam broadband satellite systems: System simulation and performance comparison with non-hopped systems. In: 5th Advanced Satellite Multimedia Systems Conference and the 11th Signal Processing for Space Communications Workshop; 2010.

7. Lejay B, et al. High power ferrite switch matrix development for advanced telecom payload at thales alenia space. 4th ESA Workshop on Advanced Flexible Telecom Payloads; 2019.

8. Bailleul PK. A new era in elemental digital beamforming for spaceborne communications phased arrays. Proc IEEE. 2016;104(13):623-632.

9. Legay H, Tubau S, Girard E, et al. Multiple beam antenna based on a parallel platewaveguide continuous delay lens beamformer. Proc Int Symp Antennas Propag. 2016:118-119.

10. Sohrabi F, Yu W. Hybrid digital and analog beamforming design for large-scale antenna arrays. IEEE J Sel Top Signal Process. 2016;10(13):501-513.

11. Tubau S, Vidal F, Legay H. Novel multiple beam antenna farms for megaconstellations; 2019.

12. Fennec H, Amos S, Hirsch A, Soumpholphakdy V. Vhts systems: requirements and evolution,; 2017:2409-2412.

13. Brélaz D. New methods to color the vertices of a graph. Commun ACM. 1979;22(14).

14. Doucet F, Fonseca NJG, Girard E, Legay H, Sauleau R. Analytical model and study of continuous parallel plate waveguide lens-like multiple-beam antennas. IEEE Trans Antenna Propagation. 2018;66(9):4426-4436.

15. Cocco G, De Cola T, Angelone M, Katona Z, Erl S. Radio resource management optimization of flexible satellite payloads for dvb-s2 systems. IEEE Trans Broadcast. 2018;64(12):266-280.

16. European electronic communications code. Off J Eur Union. 2018:12.

17. Camino JT, Mourgues S, Artigues C, Houssin L. A greedy approach combined with graph coloring for non-uniform beam layouts under antenna constraints in multibeam satellite systems. In: 7th Advanced Satellite Multimedia Systems Conference and the 13th Signal Processing for Space Communications Workshop IEEE; 2014:374-381.

18. Luo SZZQ. Dynamic spectrum management: complexity and duality. IEEE J Sel Top Signal Process. 2008;2(1):57-73.

19. Aloisio M, Angeletti P, Casini E, Colzi E, D'Addio S, Oliva-Balague R. Accurate characterization of TWTA distortion in multicarrier operation by means of a correlation-based method. IEEE Trans Electron Devices. 2009;56(5):951-958.

20. D'Addio S, Valenta V. Non-linearity assessment of SSPA-based active antennas by means of a time-domain correlation method. 2019. 4th ESA Workshop on Advanced Flexible Telecom Payloads.

21. https://artes.esa.int/projects/next-generation-high-data-rate-trunking-systems 


\section{AUTHOR BIOGRAPHIES}

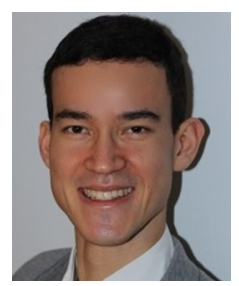

Florian Vidal received his master's degree in engineering from ISAE Supaero, Toulouse, France in 2017. In the framework of the REVOLVE project funded by the European commission, he is currently pursuing a PhD degree in electrical engineering at Heriot Watt University, Edinburgh, United Kingdom in collaboration with Thales Alenia Space, Toulouse, France and the Institut d'Electronique et de Télécommunications de Rennes, INSA Rennes, France. For 6 months, he was an intern at Inmarsat in the Space Segment Department where he carried out his master's thesis project on big data processing for satellite payload design. His current research interests include payload architectures based on multiple beam antennas and precoding technics for telecommunication satellite applications. With Thales Alenia Space, he supported the CNES study "Architectures d'antennes multifaisceaux pour constellations" to show the benefits of beam hopping to adress non-uniform user distributions.

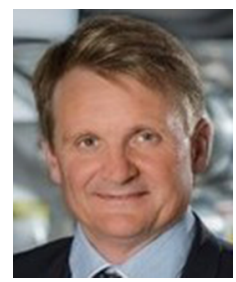

Hervé Legay received his electrical engineering and PhD degrees from the National Institute of Applied Sciences, Rennes, France, in 1988 and 1991, respectively. He was then a Post-Doctoral Fellow with the University of Manitoba, Winnipeg, MB, Canada.

In 1994, he joined Alcatel Space (now Thales Alenia Space), Toulouse, France. He initially conducted studies in the areas of military telecommunication satellite antennas and antenna processing. He designed the architecture and the antijamming process of the Syracuse 3 active antenna. He is currently the Head of the R\&T on space antennas, developing new antenna concepts with emerging technologies (Reflectarrays, integrated antennas, multiple beam quasi-optical antennas, innovative active architectures, antenna processing. He is co-director of the joint laboratory MERLIN, involving Thales Alenia Space, and the Institut d'Electronique et de Télécommunication de Rennes. Dr Legay is the chair of a group of antenna experts in Thales Group. He coordinates the collaborations with academic and research partners. He has authored 35 patents. He was a co-prize winner of the 2007 Schelkunoff Prize Paper Award. He was a recipient of the Gold Thales Awards in 2008, a reward for the best innovations in the Thales Group.

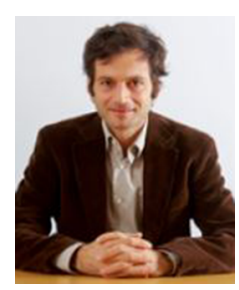

George Goussetis received his diploma degree in Electrical and Computer Engineering from the National Technical University of Athens, Greece, in 1998, and his PhD degree from the University of Westminster, London, UK, in 2002. In 2002, he also graduated BSc in physics (first class) from University College London (UCL), UK. In 1998, he joined the Space Engineering, Rome, Italy, as RF Engineer and in 1999 the Wireless Communications Research Group, University of Westminster, UK, as a research assistant. Between 2002 and 2006, he was a senior research fellow at Loughborough University, UK. He was a lecturer (assistant professor) in Heriot-Watt University, Edinburgh, UK between 2006 and 2009 and a reader (associate professor) in Queen's University Belfast, UK, between 2009 and 2012. In 2013, he joined Heriot-Watt as a reader. He has authored or co-authored over 150 peer-reviewed papers, three book chapters, and two patents. Professor Goussetis has held a research fellowship from the Onassis Foundation in 2001, a research fellowship from the UK Royal Academy of Engineering between 2006 and 2011 and a European Marie-Curie experienced researcher fellowship in 2011 to 2012. In 2010, he was a visiting professor in UPCT, Spain. He is the co-recipient of the 2011 European Space Agency young engineer of the year prize, the 2011 EuCAP best student paper prize, and the 2012 EuCAP best antenna theory paper prize.

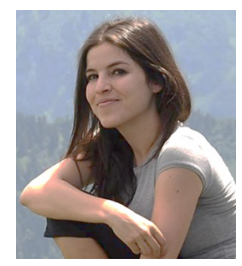

Maria Garcia Vigueras, was born in Murcia, Spain. She received both the Telecommunications Engineer and Ph.D. degrees from the Universidad Politécnica de Cartagena (UPCT), Spain, in 2007 and 2012, respectively. From 2012 to 2015, she was a Postdoctoral Research Fellow at the Laboratory of Electromagnetism and Antennas, École Polytechnique Fédérale de Lausanne (EPFL), in Switzerland. She has been a visiting researcher at Heriot-Watt University, Edinburgh, Scotland, U.K; at the University of Seville, Seville, Spain; and at Queen's University of Belfast, Belfast, Northern Ireland, U.K. Since November 2015, she works as Assistant Professor at the Institut National des Sciences Appliquées de Rennes and Researcher at IETR. Her main research interests include leaky-wave antennas (analysis, design, and pattern synthesis), periodic surfaces (physical insightful modeling), and compact antennas for satellite communications. María was the recipient of the Best Ph.D. Thesis award both from UPCT in 2013, and from the Spanish "Colegio Oficial de Ingenieros de Telecomunicaciones" (Official Association of Telecommunication Engineers) in 2014. She received the Best Antenna Theory Paper awards at the 5th IEEE Symposium on Microwave, Antenna, Propagation \& EMC Technologies (MAPE 2013, China), and the 6th European Conference of Antennas and Propagation (EuCAP 2012, Prague, Czech Republic). 


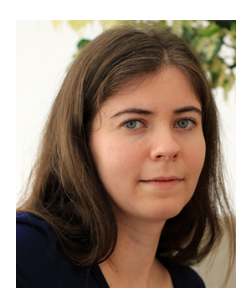

Ségolène Tubau, graduated with an electronic engineering degree from the National Institute of Electrical engineering, Electronics, Computer science, Fluid mechanics \& Telecommunications and Networks (ENSEEIHT), Toulouse, France, in 2014. She joined Thales Alenia Space in 2017 after an internship and several interim missions in this company. Her first missions included the study of new concepts of compact feeds and the development of quasi-optical beamformers during the TRP ESA Project "Lens Like Multiple Beam Antenna". Since then, she has been working on the improvement of these concepts which lead to the submission of a new patent in July 2017. She is also currently working on the adaption of quasi-optical beamformer to megaconstellations applications in the frame on a ESA

project.

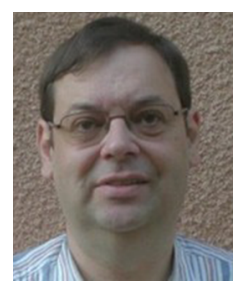

Jean-Didier Gayrard, is Senior Research Engineer in the research department of Thales Alenia Space - France in Toulouse. He is graduated in Telecommunication Engineering from Ecole Nationale Superieure de Télécommunication de Bretagne.

He jointed Alcatel Space in 1985 and started in on-board digital processor development, then in payload design and engineering. He also was in charge of payload Assembly, Integration and Test. Lastly he was in charge of satellite procurement before joining the research department in 2000.

How to cite this article: Vidal F, Legay H, Goussetis G, Garcia Vigueras M, Tubau S, Gayrard J-D. A methodology to benchmark flexible payload architectures in a megaconstellation use case. Int J Satell Commun Network. 2020;1-18. https://doi.org/10.1002/sat.1344

\section{ANNEX I: LEAST SQUARE ERROR FITTING OF MATCHING RATIO, FUNCTION OF NON-UNIFORMITY}

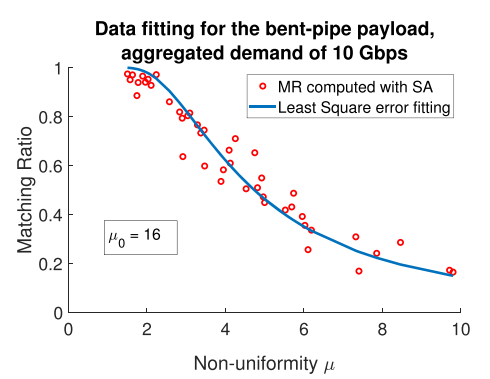

(A) Bent-pipe

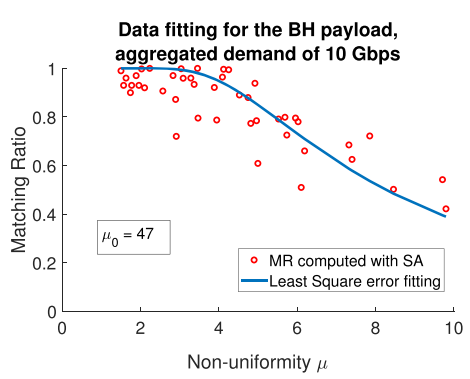

(B) Beam Hopping

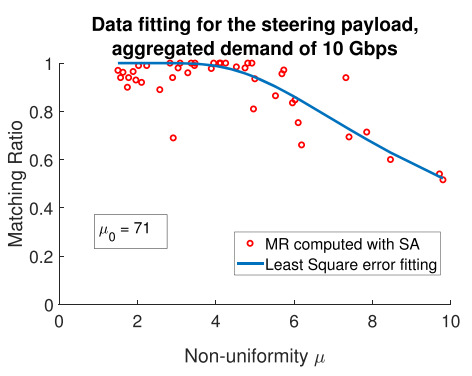

(C) Beam Steering
FIGURE A1 Least square error interpolation results with the matching ratio function model selected for A, the bent-pipe; B, beam hopping; and $C$, beam steering architectures for a total demand per scenario of $10 \mathrm{Gbps}$ [Colour figure can be viewed at wileyonlinelibrary.com] 
FIGURE A2 Least square error interpolation results with the matchign ratio function model selected for A, the bent-pipe; $B$, beam hopping; and $\mathrm{C}$, beam steering architectures for a total demand per scenario of $20 \mathrm{Gbps}$ [Colour figure can be viewed at wileyonlinelibrary.com]

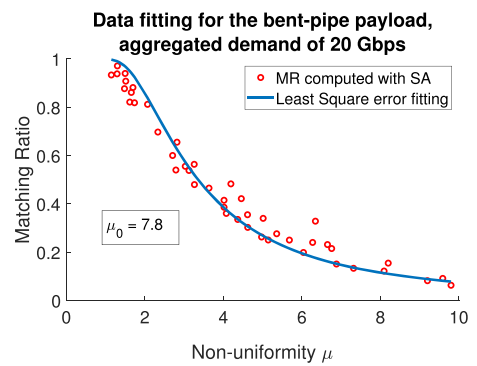

(A) Bent-pipe

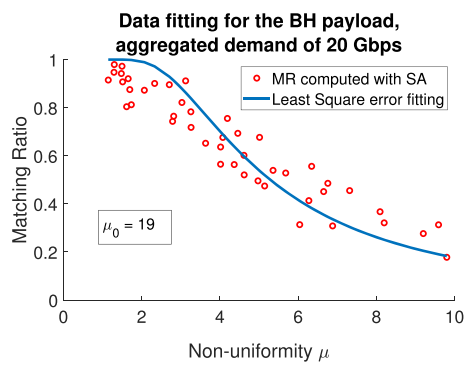

(B) Beam Hopping

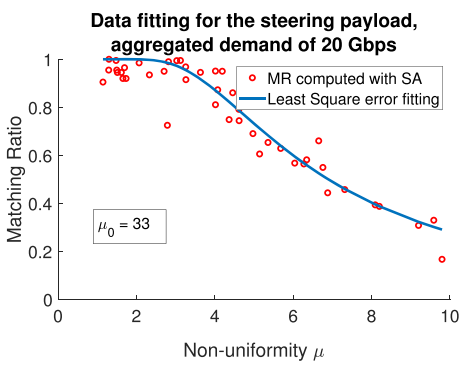

(C) Beam Steering

\section{ANNEX II: RESULTS FOR THE AERO USER DISTRIBUTION}

FIGURE B1 For the aero traffic

scenario, variation of non-uniformity, total user demand, and estimated capacity used over two 110 minute orbits for the A, bent-pipe; $B$, beam hopping; and $\mathrm{C}$, steering architectures [Colour figure can be viewed at wileyonlinelibrary.com]

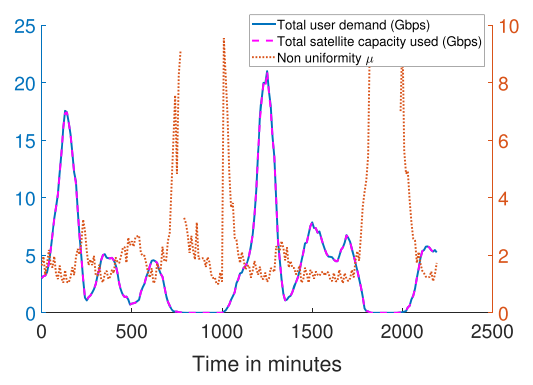

(A) Bent-pipe

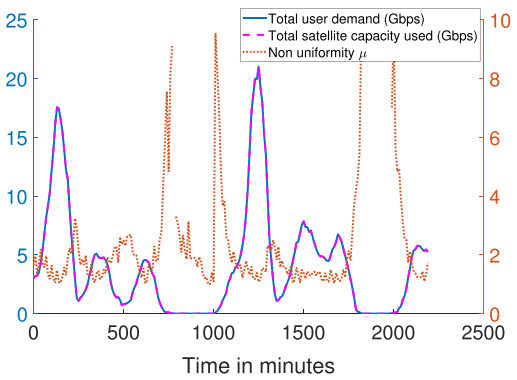

(B) Beam Hopping

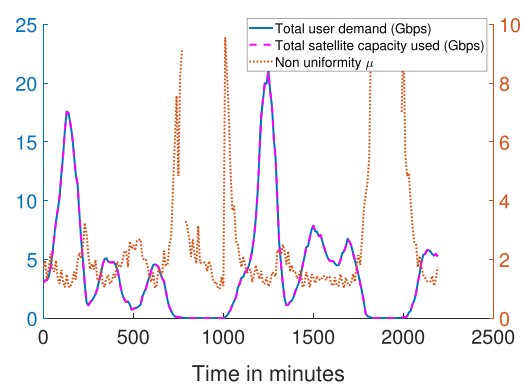

(C) Beam Steering 